\title{
DIAGNÓSTICO DE APENDICITIS EN EL TERCER TRIMESTRE DE LA GESTACIÓN: REPORTE DE CASO Y REVISIÓN DE LA LITERATURA
}

\section{Diagnosis of appendicitis in the third trimester of gestation: case report and review of the literature}

\author{
Olga Isabel Restrepo-Castro, MD MSc ${ }^{1}$; Nicolás Granados-Casallas ${ }^{2}$; \\ Carla Patricia Usta-Stavoli, MD $^{3}$; Marcos Fidel Castillo-Zamora, MD $^{4}$
}

Recibido: julio 10/17 - Aceptado: mayo 9/18

\section{RESUMEN}

Objetivo: reportar el caso de una paciente con embarazo avanzado de 35,2 semanas, con diagnóstico de apendicitis aguda con perforación y peritonitis generalizada secundaria, y hacer una revisión de la literatura publicada acerca de la utilidad de las imágenes diagnósticas en el análisis de esta condición en la gestante en la segunda mitad del embarazo.

Materiales y métodos: se presenta el caso de una paciente de 29 años, con 3 gestaciones, 2 partos, remitida a una institución de cuarto nivel de complejidad con gestación de 35,2 semanas e impresión diagnóstica de trabajo de parto pretérmino. Fue llevada a laparotomía luego de 20 horas de observación, con diagnóstico de dolor abdominal y apendicitis aguda; se encontró apendicitis perforada con peritonitis secundaria. En el posoperatorio presentó

1 Profesor Asistente de Ginecología y Obstetricia, Universidad de La Sabana, Chía (Colombia).

2 Estudiante V semestre, Facultad de Medicina, Universidad de La Sabana, Chía (Colombia).

3 Médico general, Universidad de La Sabana, Chía (Colombia).

4 Profesor Asistente de Ginecología y Obstetricia, Universidad de La Sabana, Chía (Colombia).marcoscz@unisabana.edu.co infección del sitio operatorio y trabajo de parto prematuro, por lo que se finalizó la gestación con evolución materna y perinatal satisfactorias. Se realizó una búsqueda con los términos MeSH: "Pregnancy", "Peritonitis", "Appendicitis", "Perforated Appendicitis", "Ultrasonic Diagnosis", "Magnetic Resonance Imaging”, "Computed Tomography”, en las bases de datos Up to date, Medline vía PubMed y Science Direct, para artículos publicados en inglés o español, de los últimos 20 años.

Resultados: se identificaron 20 títulos relacionados directamente con el uso de imágenes diagnósticas en mujeres gestantes con sospecha de apendicitis. El ultrasonido es la tecnología diagnóstica utilizada como primera opción, sin embargo, su desempeño diagnóstico en el segundo y tercer trimestre es limitado porque frecuentemente no se logra visualizar el apéndice. La sensibilidad varía del 46 al 63\%, y la especificidad del 80 al 100\%; la resonancia magnética tiene un mejor desempeño operativo, con una sensibilidad que varía entre el 60 y el $100 \%$, y una especificidad del 95 al $100 \%$, aunque tiene restricciones por ser más costosa y tener limitaciones de acceso. 
Conclusiones: el diagnóstico de la apendicitis aguda en el embarazo es un reto diagnóstico, la resonancia magnética podría ser más útil que el ultrasonido para el diagnóstico en el segundo y tercer trimestre de embarazo.

Palabras clave: peritonitis, apendicitis, embarazo, diagnóstico ultrasonido.

\section{ABSTRACT}

Objective: To report the case of a woman in the third trimester of pregnancy diagnosed with perforated acute appendicitis and secondary generalised peritonitis; and to review the published literature on the usefulness of diagnostic imaging as part of the workup for this condition during the second half of pregnancy.

Materials and methods: We present the case of a 29-year-old patient, gravida 3 para 2, referred to a high complexity institution at 35.2 weeks of gestation with a diagnostic impression of pre-term labour. The patient was taken to laparotomy after remaining under observation for 20 hours of observation, with a diagnosis of abdominal pain and acute appendicitis, and was found to have perforated appendicitis with secondary peritonitis. Post-operatively, the patient developed surgical site infection and premature labour, leading to preterm delivery with satisfactory maternal and perinatal outcome. A search for articles published in English or Spanish over the past 20 years was conducted in the Up to date, Medline vía PubMed and Science Direct databases using MeSH terms "Pregnancy," "Peritonitis," "Appendicitis", "Perforated Appendicitis," "Ultrasonic Diagnosis," "Magnetic Resonance Imaging," "Computed Tomography."

Results: Overall, 20 titles directly related to the use of diagnostic imaging in pregnant women with suspected appendicitis were identified. Ultrasound is the first option used for diagnosis, but its diagnostic accuracy during the second and third trimesters is limited because, frequently, it is not possible to visualise the appendix. Sensitivity varies between $46 \%$ and $63 \%$, and specificity between $80 \%$ and $100 \%$.
Nuclear magnetic resonance has better operational performance, with sensitivity ranging between 60\% and $100 \%$, and specificity ranging between $95 \%$ and $100 \%$, although it is more expensive and has access limitations.

Conclusions: The diagnosis of acute appendicitis in pregnancy is challenging. Nuclear magnetic resonance would be more useful than ultrasound for diagnosis during the second and third trimesters.

Key words: peritonitis, appendicitis, pregnancy, ultrasound diagnosis.

\section{INTRODUCCIÓN}

Dentro de las causas de abdomen agudo que afectan a la mujer embarazada encontramos en la literatura como la más común la inflamación del apéndice secundaria a infección o apendicitis aguda (1), con una frecuencia de 0,4 a 1,4 por cada 1000 embarazos (2); esta causa el $25 \%$ de las cirugías no obstétricas durante la gestación (3).

El diagnóstico oportuno es crítico para evitar la perforación del apéndice, sin embargo, el embarazo representa un reto especial en esta condición. Aunque la sintomatología es similar a la de la mujer no gestante-caracterizada por dolor abdominal agudo que inicia en el epigastrio o región periumbilical que irradia a fosa iliaca derecha, con frecuencia asociado a fiebre, vómito y taquicardia-, se ha descrito que los signos clínicos clásicos tienen menor exactitud en las gestantes por los cambios anatómicos tales como el crecimiento uterino en un embarazo avanzado, el cual hace que el epiplón, el intestino delgado y la pared abdominal se desplacen y se alejan del apéndice cecal (4). Dado que la ubicación del apéndice varía de acuerdo con el tiempo de gestación, en el primer trimestre tendrá un desplazamiento cefálico sobre el punto de McBurney, la migración continuará hasta el octavo mes donde se ubicará en el $80 \%$ de las ocasiones en la región subcostal derecha (5). Este desplazamiento puede causar un retraso diagnóstico y aumento en la frecuencia de perforación (6).

Se ha descrito que la perforación del apéndice durante el embarazo se acompaña de mayor mor- 
bilidad materna (52\% vs. $17 \%$ sin perforación) y mayor mortalidad que puede alcanzar hasta el $4 \%$ (7). Por otra parte, se ha descrito que la apendicitis aguda perforada lleva a un incremento de actividad uterina pretérmino y parto pretérmino (8), y la mortalidad fetal se presenta en un $24 \%$ cuando se perfora el apéndice, comparado con un $7 \%$ cuando no hay perforación $(9,10)$.

Como se mencionó, el diagnóstico de apendicitis en el embarazo representa un reto tanto para el obstetra como para el cirujano y el médico general. En la actualidad hay ayudas diagnósticas tales como el ultrasonido y la resonancia magnética nuclear (RMN), que deberían facilitar el diagnóstico temprano de la apendicitis en el embarazo, por lo que es importante conocer la calidad de la evidencia que soporta su uso en esta población, para seleccionar la mejor ayuda diagnóstica. El objetivo de la presentación de este caso de apendicitis en la segunda mitad del embarazo es hacer una revisión de la literatura del abordaje diagnóstico dado a la apendicitis aguda en el embarazo avanzado, con énfasis en la utilidad del ultrasonido y la resonancia magnética nuclear.

\section{PRESENTACIÓN DEL CASO}

Paciente de 29 años, con gestación de 35,2 semanas por fecha de última menstruación, de estado civil soltera, ama de casa, de raza mestiza, quien ingresó al hospital de cuarto nivel de complejidad en la ciudad de Bogotá (Colombia) remitida por una institución de primer nivel de complejidad por cuadro clínico de un día de evolución. La paciente consultó a esta última institución por dolor epigástrico, el cual no cedió con la administración de antagonista de los receptores de histamina; en los paraclínicos, el hemograma evidenció leucocitosis y neutrofilia con uroanálisis normal. Se consideró amenaza de parto pretérmino, razón por la cual se decidió la remisión (figura 1). Al ingreso la paciente refirió dolor a nivel de hipogastrio sin otra sintomatología asociada, con único antecedente patológico de importancia de asma en manejo con $\beta 2$ agonista; en los antecedentes obstétricos existen dos gestaciones previas sin complicaciones. Actual gestación con STORCH (sífilis, toxoplasma, rubeola, citomegalovirus, herpes, hepatitis B) negativo, ecografías de primer y segundo trimestre concordantes para la edad gestacional, con único dato relevante de la primipaternidad.

$\mathrm{Al}$ examen físico de ingreso se encontró tensión arterial: 115/66 mm/Hg, frecuencia cardiaca: 97 latidos por minuto, frecuencia respiratoria: 18 respiraciones por minuto, temperatura: $36,5{ }^{\circ} \mathrm{C}$, saturación arterial de oxígeno: 95 \%; con dolor a la palpación de hemiabdomen inferior y evidencia de actividad uterina irregular, por lo que se consideró distensión ligamentaria y se dio manejo expectante. Concomitante, se solicitó hemograma que evidenció 15.900 leucocitos $/ \mathrm{mm}^{3}$ ), a expensas de neutrófilos, PCR (proteína C reactiva) elevada, función hepática normal y uroanálisis no sugestivo de infección. Después de 20 horas, la paciente presentó signos clínicos de respuesta inflamatoria sistémica dados por pico febril y taquicardia, con dolor a nivel de flanco derecho y defensa voluntaria, además de monitoreo fetal con taquicardia fetal, el cual se explicó por fiebre materna. Los paraclínicos de control evidenciaron elevación de reactantes de fase aguda con persistencia de PCR elevada y aumento de leucocitosis.

Dada la sospecha de apendicitis aguda fue valorada por el servicio de cirugía general, y ante la posibilidad de patología quirúrgica abdominal realizaron laparotomía exploratoria mediana donde se evidenció apendicitis aguda en fase gangrenosa, perforada en tercio medio, con peritonitis generalizada; se realizó apendicectomía más lavado peritoneal y se inició antibioticoterapia con piperacilina/ tazobactam 4,5 mg intravenoso cada 6 horas, más metronidazol $500 \mathrm{mg}$ intravenoso cada 8 horas.

Durante su posoperatorio se evidenció aumento de eritema perilesional en herida quirúrgica y, adicionalmente, la paciente refirió actividad uterina por lo que se realizó examen ginecológico que mostró cambios cervicales, dilatación de $3 \mathrm{~cm}$ con borramiento del $70 \%$, y monitoreo fetal con actividad uterina 


\section{Figura 1.}

Cronología del estado de la paciente durante la estancia hospitalaria

PACIENTE DE 29 AÑOS MULTIGESTANTE CON EMBARAZO DE 35.2

SEMANAS QUIEN CONSULTA POR DOLOR ABDOMINAL

Paciente consultó a centro de salud de primer nivel de complejidad por epigastralgia. Se da egreso con manejo analgésico.

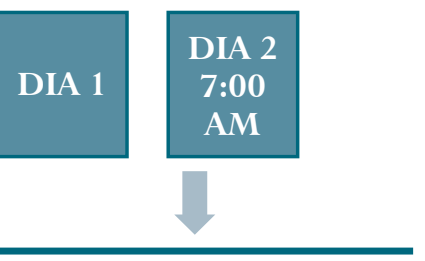

Paciente reconsultó; toma de paraclínicos que evidencian leucocitos y neutrofila. Se decide remitir por sospecha de amenaza de parto pretermino a un centro de salud de mayor nivel de complejidad.
Al ingreso paciente refirió dolor en hipogastrio y al examen físico taquicardia materna por lo que se solicitan paraclínicos y se indica manejo expectante.

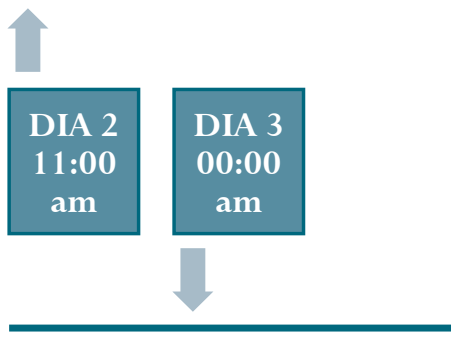

Se evidencia signos de respuesta inflamatoria sistémica, dolor en flanco derecho; con reactantes de fase aguda elevados. Se consideró apendicitis aguda y se realizó laparotomía exploratoria en la que se encontró peritonitis generalizada.
Mejoría clínica.

Paciente refiere actividad uterina regular, con cambios cervicales y adicionalmente se evidenció infección de sitio operatorio superficial. Se indica finalización de la gestación por vía abdominal.

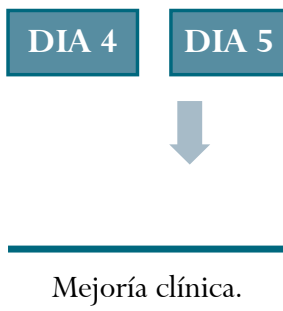

DIA 6

DIA (7-15)

Posterior a la segunda intervención quirúrgica se evidenció resolución de cuadro infeccioso, se continuó terapia antibiótica por 7 días más y se dio egreso.

Fuente: los autores.

irregular. Cirugía general revaloró a la paciente y consideró infección de sitio operatorio superficial complicada, por lo que indicaron reintervención quirúrgica para manejo de herida quirúrgica abierta; durante el procedimiento se evidencia dehiscencia de la fascia, por lo que se consideró realizar valoración intraoperatoria por el servicio de ginecoobstetricia que decide, con base en los hallazgos de un proceso infeccioso activo en cavidad abdominal y riesgo de sepsis materna con compromiso fetal, finalizar el embrazo en el mismo acto quirúrgico por medio de una cesárea. Ambos procedimientos se realizaron sin complicaciones, con obtención de recién nacido vivo de sexo masculino, peso de $3340 \mathrm{~g}$, talla de 48 cm con APGAR bajo recuperado. Madre e hijo permanecieron en hospitalización hasta que se finalizó esquema antibiótico de 7 días.

\section{MATERIALES Y MÉTODOS}

Diseño y población. Se llevó a cabo una búsqueda de la literatura en las bases de datos Up to date, Medline vía PubMed y Science Direct, con la terminología MeSH "Pregnancy", "Peritonitis", "Appendicitis", "Perforated Appendicitis", "Ultrasonic Diagnosis", "Magnetic Resonance Imaging" y "Computed Tomography”. Se realizó una búsqueda en la que se incluyeron reportes de caso, revisiones de la literatura, estudios descriptivos retrospectivos, cohortes, casos y controles publicados en idioma inglés o español, de los últimos 20 años, que se centraran en apendicitis en el embarazo avanzado, y en los que se abordara el enfoque diagnóstico. Se excluyeron cartas al editor.

Aspectos éticos. La paciente firmó el consentimiento informado y dio autorización para la publicación de 
su caso. Se garantizaron la confidencialidad de la información y la privacidad.

\section{RESULTADOS}

Se identificaron 10 títulos relativos al uso de imágenes para el diagnóstico de apendicitis en el embarazo, de los cuales tres correspondieron a estudios de cohortes retrospectivas (11-13), cinco a estudios de corte transversal (14-18), una cohorte prospectiva (19) y una revisión de la literatura (20). Los estudios fueron realizados en Estados Unidos (12-15), Canadá (17, 18), Brasil (20), Turquía (11), Irán (19) y Corea del Sur (16).

Ultrasonido. Franca et al., en su revisión de la literatura, plantean que el abordaje diagnóstico de la apendicitis en sus estadios iniciales resulta difícil de realizar en mujeres embarazadas, especialmente en el segundo y tercer trimestre del embarazo; asimismo, indican que el primer estudio diagnóstico sería el ultrasonido, y recomiendan la resonancia magnética nuclear cuando este no es concluyente y, como último recurso, se plantea el uso de la tomografía axial computarizada (20).

Aras et al., en un estudio de cohorte retrospectiva de mujeres gestantes y no gestantes sometidas a apendectomía informa que el ultrasonido en el diagnóstico apendicitis en la mujer gestante tiene una sensibilidad del $61 \%$ y una especificidad del $80 \%$. Sugiere una evaluación cuidadosa en aquellas pacientes con sospecha de apendicitis en las que la ultrasonografía es reportada como no concluyente o normal, debido a que en las pacientes embarazadas en el tercer trimestre de gestación, el dolor puede estar localizado en el cuadrante superior derecho y suele haber un aumento ligero de los leucocitos en esta etapa de la gestación lo que puede generar una limitación diagnóstica (11).

Kazemini et al., en un estudio de cohorte prospectiva, evaluaron la exactitud de la ultrasonografía en el diagnóstico de la apendicitis aguda en el embarazo. Estudiaron 58 mujeres embarazadas con edad promedio de 29,1 \pm 4,94 con diagnóstico de apendicitis aguda confirmado histológicamente, entre enero del 2014 y enero del 2016. Informan que la sensibilidad disminuye a mayor edad gestacional mientras que la especificidad aumenta; reportan una sensibilidad del $63 \%$ en el segundo trimestre y del $50 \%$ en el tercer trimestre, una especificidad del 75 y $100 \%$ respectivamente, y una razón de probabilidades positiva del 2,52 y negativa de 0,49 para el segundo trimestre. Este autor recomienda el uso de otras imágenes tales como tomografía computarizada o resonancia magnética después de un resultado ultrasonográfico no concluyente (19).

Shetty et al. realizaron un estudio de corte transversal retrospectivo en un periodo de 5 años en historias clínicas, enfocándose en imágenes diagnósticas realizadas a pacientes que tenían una sospecha clínica de apendicitis; se correlacionaron los hallazgos imagenológicos con el manejo de la paciente y el desenlace final. Un total de 39 pacientes embarazadas fueron remitidas para estudio de imágenes diagnósticas, de estas, 35 fueron sometidas a una evaluación ecográfica, 23 de estas mujeres fueron examinadas posteriormente con tomografía axial computarizada y 4 pacientes fueron directamente evaluadas con tomografía sin haber sido sometidas a ecografía previamente; concluyen que el método diagnóstico más utilizado es la ultrasonografía, con una sensibilidad del 46,1\% y una especificidad 95,4\%. La baja sensibilidad se podría explicar porque frecuentemente no hay visualización del apéndice (14).

Resonancia magnética nuclear. Theilen et al., en un estudio de cohorte retrospectiva, evaluó la exactitud de la resonancia magnética para el diagnóstico de apendicitis en 171 mujeres embarazadas con sospecha de esta inflamación. Informan que en 53 gestantes no se pudo visualizar el apéndice y ninguna presentó finalmente apendicitis. En las que el diagnóstico fue de apéndice normal solo una tuvo apendicitis, y en las 18 que tuvieron diagnóstico de apéndice anormal hubo 6 falsos positivos; de esta manera, la RM mostró una sensibilidad del 91 \% y una especificidad del 95,3\%. Los autores mencionan además que entre mayor sea el trimestre más se disminuye 
la tasa de visualización, pero aun así es mejor que la ultrasonografía, que se realizó en 46 de las mujeres gestantes con no visualización en 43 de ellas. De las 3 en las que se pudo visualizar, en 2 se diagnosticó apéndice anormal y se confirmó solo en una, así como en la que se consideró normal. De las 43 pacientes en las que no se visualizó el apéndice, en 28 se logró el diagnóstico por RM posteriormente (12).

Israel et al., en un estudio de cohorte retrospectiva donde se incluyeron 33 pacientes con sospecha de apendicitis, en las cuales se confirmaron 5 casos de apendicitis, la resonancia identificó como apéndice anormal los 4 casos de apendicitis aguda, en 13 se hizo el diagnóstico de apéndice normal, en ninguno se identificó el diagnóstico de apendicitis en la historia clínica, y en 16 no se pudo identificar el apéndice, uno de los cuales resultó en apendicitis crónica. De esta manera, la sensibilidad fue del $80 \%$ y la especificidad del 100\%. Estos autores reportan que el ultrasonido no identificó el apéndice en 29 gestantes, en 3 el diagnóstico fue de apéndice normal, uno de los cuales fue diagnosticado finalmente con apendicitis aguda. Los autores no informan la edad gestacional a la que se hicieron las imágenes diagnósticas (13).

Tsai et al. realizaron un estudio de corte transversal retrospectivo que buscaba hallar el grado de concordancia interradiólogo frente a las características de la resonancia magnética en la apendicitis durante el embarazo, junto con los resultados asociados a una interpretación indeterminada. Estudiaron 233 mujeres con sospecha de apendicitis en el embarazo entre 2003 y 2015, las cuales fueron sometidas a resonancia magnética durante un periodo. En total hubo 14 pacientes (6\%) positivas para la apendicitis aguda durante el embarazo, en 13 de estas pacientes la RM fue interpretada como anormal y en una paciente como normal; la concordancia interobservador tuvo un kappa de 0,85 a 1; en 73 pacientes donde el apéndice no pudo ser visualizado, ninguna tuvo como resultado final apendicitis (15).

Jung et al. llevaron a cabo un estudio de corte transversal retrospectivo para evaluar la exactitud diagnóstica de la RM en apendicitis, se realizó en 46 pacientes embarazadas que fueron sometidas a resonancia magnética del apéndice con sospecha de apendicitis aguda entre 2010 y 2016; la RM mostró una sensibilidad del $100 \%$ y una especificidad del $91 \%$, este examen permitió categorizar en apendicitis probable sola, apendicitis asociada a otra patología pélvica y diagnóstico definitivo de apendicitis. Dos de los tres falsos positivos de la RM fueron en diagnóstico probable de apendicitis acompañado de otra patología (16).

Patel et al., en otro estudio de corte transversal retrospectivo en 42 pacientes embarazadas con sospecha de apendicitis, a quienes se realizó ultrasonido y luego resonancia magnética entre agosto de 2008 y 2015, buscaron determinar la exactitud diagnóstica de los 42 exámenes de resonancia magnética. En total hubo 5 pacientes con diagnóstico de apendicitis aguda y se realizaron 7 apendicectomías. El ultrasonido no identificó el apéndice en ninguna paciente, mientas que la RM lo identificó en 22, que se calificaron como apendicitis en 6 y normales en 16. Hubo 20 casos en los que no se visualizó el apéndice, de los cuales en ninguno se diagnosticó apendicitis en los 6 meses siguientes. Finalmente, la RM identificó adecuadamente 3 casos de apendicitis aguda, 3 se consideraron falsos positivos y en 36 clasificados como apéndice normal hubo 34 verdaderos negativos y dos falsos negativos, para una sensibilidad del $60 \%$ y una especificidad del $92 \%$ (17).

Burns et al., en un estudio de corte transversal retrospectivo, buscaron evaluar el rendimiento de RM para el diagnóstico de apendicitis durante el embarazo en una institución canadiense; se realizó una revisión de todas las imágenes por RM realizadas entre 2006 y 2012, para la evaluación de sospecha de apendicitis en mujeres embarazadas; se revisaron un total de 71 resonancias; el apéndice fue identificado en 40 pacientes (56,3\%), la sensibilidad de la RM fue del 75 \% y la especificidad del $100 \%$ para el diagnóstico de apendicitis en mujeres embarazadas (18). 


\section{CONCLUSIONES}

El diagnóstico de la apendicitis en el embarazo es difícil, especialmente en el segundo y tercer trimestre de la gestación, por el desplazamiento cefálico del apéndice. A pesar de que el ultrasonido es considerado como primera opción por la facilidad en su realización y su bajo costo tiene una sensibilidad entre el 46 y $63 \%$, y una especificidad del 85 y el $100 \%$. La sensibilidad es menor a mayor edad gestacional, época en que a menudo no se puede visualizar el apéndice. La resonancia magnética es un método más costoso y demorado, con una sensibilidad del 60 al $100 \%$ y una especificidad del 91 al $100 \%$. Esta técnica diagnóstica sería más útil en el segundo y tercer trimestre de gestación. En los estudios incluidos no hubo casos de apendicitis cuando no se visualizó el apéndice.

\section{REFERENCIAS}

1. Medilexicom: Acute Appendicitis. Definition [visitado 2018 mar 5]. Disponible en: http://www.medilexicon. com/dictionary/5696.

2. Bigot GS. Apendicitis aguda y embarazo. Rev Méd Costa Rica Centroamérica. 2008;361-4.

3. Arer IM. Acute appendicitis during pregnancy: Case series of 20 pregnant women. TJTES. 2016;22:545-8. https://doi.org/10.5505/tjtes.2016.58458

4. Laffita Labañino W, Jiménez W. Apendicitis aguda en el embarazo. Rev Cubana Obstet Ginecol. 2011 [cited 2018 mar 21];37(2):223-34. Available in: http://scielo. sld.cu/scielo.php?script $=$ sci_arttext\&pid $=$ S0138600X2011000200012\&lng =es

5. Sabiston D, Townsend C. Sabiston Tratado de cirugía. Barcelona: Elsevier; 2013.

6. Terzi A, Yildiz F, Vural M, Coban S, Cece H, Kaya M. A case series of 46 appendectomies during pregnancy. Wien Klin Wochenschr. 2010;122:686-90. https:// doi.org/10.1007/s00508-010-1492-0.

7. Yilmaz HG, Akgun Y, Bac B, Celik Y. Acute appendicitis in pregnancy - Risk factors associated with principal outcomes: A case control study. Int J Surg. 2007;5:1927. https://doi.org/10.1016/j.ijsu.2006.05.005

8. Mourad J, Elliott JP, Erickson L, Lisboa L. Appendicitis in pregnancy: New information that contradicts long-held clinical beliefs. Am J Obstet Gynecol.
2000;182:1027-9. https://doi.org/10.1067/ mob.2000.105396.

9. Humes DJ, Simpson J. Acute appendicitis. BMJ. 2006;333:530-4. https://doi.org/10.1136/bmj. 38940.664363.AE

10. Sharma A, Sharma R, Sharma S. Appendicitis in pregnancy: A novel approach for diagnostic dilema. SLJOG. 2012;6-10.

11. Aras A, Karaman E, Pekşen Ç, Kiziltan R, Çetin M. The diagnosis of acute appendicitis in pregnant versus nonpregnant women: A comparative study. Rev Assoc Med Bras. 2016;62:622-7. https://doi.org/10.1590/18069282.62.07.622

12. Theilen LH, Mellnick VM, Longman RE, Tuuli MG, Odibo AO, MacOnes GA, et al. Utility of magnetic resonance imaging for suspected appendicitis in pregnant women. Am J Obstet Gynecol. 2015; 212:345.e1-345.e6. https://doi.org/10.1016/j. ajog.2014.10.002.

13. Israel G, Malguria N, McCarthy S, Copel J, Weinreb J. MRI vs. ultrasound for suspected appendicitis during pregnancy. J Magn Reson Imaging. 2008;28:428-33. https://doi.org/10.1002/jmri.21456.

14. Shetty MK, Garrett NM, Carpenter WS, Shah YP, Roberts C. Abdominal computed tomography during pregnancy for suspected appendicitis: 5 -year experience at a maternity hospital. Seminars in Ultrasound, CT and MRI. 2010;31:8-13. https://doi. org/10.1053/j.sult.2009.09.002.

15. Tsai R, Raptis C, Fowler KJ, Owen JW, Mellnick VM. MRI of suspected appendicitis during pregnancy: Interradiologist agreement, indeterminate interpretation and the meaning of non-visualization of the appendix. Br J Radiol. 2017;90:1079. https://doi.org/10.1259/ bjr.20170383.

16. Jung JY, Na JU, Han SK, Choi PC, LEE JH, Shin DH. Differential diagnoses of magnetic resonance imaging for suspected acute appendicitis in pregnant patients. World J Emerg Surg. 2018;9:26-32. https:// doi.org/10.5847/wjem.j.1920-8642.2018.01.004

17. Patel D, Fingard J, Winters S, Low G. Clinical use of MRI for the evaluation of acute appendicitis during pregnancy. Abdom Radiol. 2017;42:1857-63. https:// doi.org/10.1007/s00261-017-1078-7.

18. Burns M, Hague CJ, Vos P, Tiwari P, Wiseman SM. Utility of magnetic resonance imaging for the diagnosis of appendicitis during pregnancy: A canadian 
experience. Can Assoc Radiol J. 2017;68:392-400. https://doi.org/10.1016/j.carj.2017.02.004.

19. Kazemini A, Reza Keramati M, Fazeli MS, Keshvari A, Khaki S, Rahnemai-Azar A. Accuracy of ultrasonography in diagnosing acute appendicitis during pregnancy based on surgical findings. Med J Islam Repub Iran. 2017;31:48. https://doi.org/10.14196/mjiri.31.48.
20. Franca Neto AH, Amorim MM, Nobrega BM. Acute appendicitis in pregnancy: Literature review. Rev Assoc Med Bras. 2015;61:170-7. https://doi. org/10.1590/1806-9282.61.02.170.

\section{Conflicto de intereses: ninguno declarado.}

